\title{
Dynamic Factor Model with infinite-dimensional factor space: Forecasting
}

\author{
Mario Forni, Alessandro Giovannelli, Marco LipPI, \\ Stefano SocCORsI *
}

April 12, 2018

\begin{abstract}
The paper compares the pseudo real-time forecasting performance of three Dynamic Factor Models: (i) The standard principal-component model introduced by Stock and Watson in 2002, (ii) The model based on generalized principal components, introduced by Forni, Hallin, Lippi and Reichlin in 2005, (iii) The model recently proposed by Forni, Hallin, Lippi and Zaffaroni in 2015. We employ a large monthly dataset of macroeconomic and financial time series for the U.S. economy, which includes the Great Moderation, the Great Recession and the subsequent recovery (an update of the so-called Stock and Watson dataset). Using a rolling window for estimation and prediction, we find that (iii) significantly outperforms (i) and (ii) in the Great Moderation period for both Industrial Production and Inflation, that (iii) is also the best method for Inflation over the full sample. However, (iii) is outperformed by (ii) and (i) over the full sample for Industrial Production.

Keywords: Time Series Forecasting, Macroeconomic Forecasting, Dynamic Factor Models.

*Forni: Università di Modena e Reggio Emilia, Modena, Italy, CEPR and RECent. Giovannelli: Università di Roma Tor Vergata, Roma, Italy. Lippi (Corresponding author) Einaudi Institute for Economics and Finance, Via Sallustiana 62, 00187 Roma, Italy. Email: mlippi.eief@gmail.com. Soccorsi: Lancaster University Management School, Lancaster, UK. We are grateful for very useful suggestions to Donatella Albano, Manfred Deistler, Fabio Della Marra, Domenico Giannone, JeanPaul L'Huillier and two anonymous referees. The contribution of the Italian Ministry of Education, University and Research for the PRIN project 2010J3LZEN_003 is gratefully acknowledged.
\end{abstract}




\section{Introduction}

This paper compares the pseudo real-time forecasting performance of three LargeDimensional Dynamic Factor Models for the US monthly macroeconomic dataset over the period February 1985 to August 2014, this including the so-called Great Moderation and the Great Recession.

Large-Dimensional Dynamic Factor Models represent each variable in the dataset as decomposed into a common component, driven by a small (as compared to the number of series in the dataset) and fixed (as the number of series grows) number of common factors and an idiosyncratic component. The latter are assumed to be orthogonal across different variables or only weakly correlated, so that the covariance of the variables is mostly accounted for by the common components. Typically, the asymptotic results are obtained for $n$, the number of series, and $T$, the number of observations for each series, both tending to infinity. Among the different versions of the Dynamic Factor Model we selected:

(i) SW. The model introduced in Stock and Watson (2002a,b). The factors are estimated by means of the standard Principal Components of the variables in the dataset. The forecast of the variable of interest, call it $y_{t}$, is obtained by regressing $y_{t+h}$ on the factors and the variable $y_{t}$, plus possibly lags of the factors and $y_{t}$.

(ii) FHLR. A variant of the previous model which has been proposed in Forni et al. (2005). In a first step the covariances of the common and the idiosyncratic components are estimated using a frequency-domain method introduced in Forni et al. (2000). In the second step such covariances are employed to estimate the factors by means of Generalized Principal Components.

(iii) FHLZ. Both models (i) and (ii) assume that the space spanned by the common components at any time $t$ stays finite-dimensional as $n$ tends to infinity. In two recent papers, Forni et al. $(2015,2017)$, it is assumed that a finite number of common shocks drive the common components, though the common components themselves are allowed to span an infinite-dimensional space. The 
dynamic relationship between the variables and the factors in this model is more general as compared to (i) and (ii). However, its estimation is rather complex and no systematic comparison with (i) and (ii) has as yet been produced.

The literature comparing SW and FHLR has reached mixed conclusions so far. Using the monthly U.S. macroeconomic dataset known as the Stock and Watson dataset, Boivin and $\mathrm{Ng}$ (2005) found that SW generally outperforms FHLR, whereas D'Agostino and Giannone (2012) found the two methods to perform equally well in their sample, even if different performances are found in subsamples. In particular, FHLR fares better during the Great Moderation, consistently with the results in the present paper. Schumacher (2007), using German data, finds that FHLR provides more accurate forecasts of the GDP. A similar result is obtained in den Reijer (2005) with Dutch macroeconomic data.

In the present paper we extend the comparisons in Boivin and $\mathrm{Ng}$ (2005) and D'Agostino and Giannone (2012) to an update of the Stock and Watson dataset, and include the new FHLZ forecasting model. Our dataset starts in January 1959 and end in August 2014, thus including the Great Moderation, the Great Recession and the subsequent recovery.

The main task of the paper is evaluating the performance of the new model FHLZ with respect to SW, the standard in this literature, and FHLR, which shares with FHLZ the frequency domain approach. Important variants of the Dynamic Factor Model such as e.g. Peña and Poncela (2004), Kapetanios and Marcellino (2009) are not considered (for wider comparisons of forecast results with Dynamic Factor Models, see Schumacher (2007) and Eickmeier and Ziegler (2008)). Rather, we compare FHLZ, FHLR and SW with (1) two "second-generation" factor models, namely Doz et al. (2011), in which a maximum likelihood estimation method of the factors is introduced, and the three-pass regression filter of Kelly and Pruitt (2015), (2) a model based on Bayesian shrinkage, De Mol et al. (2008).

A distinctive feature of our exercise is that we use a fairly large subsample, February 1960 to December 1984, to calibrate the models. In particular, how to determine the number of factors in SW and FHLR, the number of lags of the factors 
or of the variable to be predicted, the Kernel in the spectral estimation for FHLR and FHLZ, etc. The selected models are then run and compared in the remaining sample, January 1985 to August 2014, which makes the present paper the first to provide a thorough comparison of different factor models over the Great Moderation, the Great Recession and the subsequent recovery.

Our main results are:

(I) In the Great Moderation period, where the assumption of stationarity of the series in the dataset (after suitable transformations) underlying all factor models is by and large fulfilled, FHLZ significantly outperforms FHLR, SW and AR both for Industrial Production and Inflation.

(II) In the full sample, including the Great Recession and the subsequent recovery, FHLZ remains the best method for CPI, albeit slightly, whereas FHLR and SW outperform FHLZ and AR for Industrial Production.

(III) We also run forecasts for all single series in the dataset over the full sample. Consistently with (II) above, FHLZ is the best method for the nominal variables whereas FHLR is the best for real variables.

The structure of the paper is as follows. In Section 2 the models SW, FHLR and FHLZ are outlined and the particular features of FHLZ are discussed. In Section 3 we describe the calibration of the models. In Section 4 we present and discuss the main results. Section 5 concludes. Some features of the forecasting models, details of the calibration procedure and additional empirical results have been gathered in the Appendix (not for publication).

\section{Three forecasting methods}

Let us start with the general form of the Large-Dimensional Dynamic Factor Model:

$$
x_{i t}=\chi_{i t}+\xi_{i t}=\frac{c_{i 1}(L)}{d_{i 1}(L)} u_{1 t}+\frac{c_{i 2}(L)}{d_{i 2}(L)} u_{2 t}+\cdots+\frac{c_{i q}(L)}{d_{i q}(L)} u_{q t}+\xi_{i t} \text {, }
$$


where $L$ is the lag operator, $t \in \mathbb{Z}, i \in \mathbb{N}$,

$$
c_{i f}(L)=c_{i f, 0}+c_{i f, 1} L+\ldots+c_{i f, s_{1}} L^{s_{1}}, \quad d_{i f}(L)=1+d_{i f, 1} L+\ldots+d_{i f, s_{2}} L^{s_{2}},
$$

$f=1,2, \ldots, q, \mathbf{u}_{t}=\left(u_{1 t} u_{2 t} \cdots u_{q t}\right)^{\prime}$ is a $q$-dimensional orthonormal white noise. The processes $\chi_{i t}$, are called the common components, they are driven by the common shocks $\mathbf{u}_{t}$, also called the dynamic (common) factors. We assume that the polynomials $d_{i f}(L)$ are stable so that $\chi_{i t}$ is stationary and is co-stationary with $\chi_{j t}$ for all $i, j \in \mathbb{N}$. The processes $\xi_{i t}$ are called the idiosyncratic components. We assume that $\xi_{i t}$ is stationary and co-stationary with $\xi_{j t}$ for all $i, j \in \mathbb{N}$. Moreover, $\xi_{i t}$ and $\mathbf{u}_{t}$ are orthogonal for all $i \in \mathbb{N}$ so that $\xi_{i t}$ and $\chi_{j t}$ are orthogonal for all $i, j \in \mathbb{N}$. The assumptions above imply that the process $x_{i t}$ is stationary and costationary with $x_{j t}$, for all $i, j \in \mathbb{N}$. Only the processes $x_{i t}$ are supposed to be observable, the components $\chi_{i t}$ and $\xi_{i t}$, the shock vector $\mathbf{u}_{t}$ and its dimension $q$, are unobserved and must be estimated. Moreover, though we suppose that the common components have a VARMA structure, we place no restrictions on the rational functions in (2.1).

Assumptions on the covariances $\mathrm{E}\left(\chi_{i t} \chi_{j t}\right)$ and $\mathrm{E}\left(\xi_{i t} \xi_{j t}\right)$ ensure that linear combinations of the idiosyncratic components, with coefficients sufficiently well spread across the variables, tend to zero in variance, whereas those of the common components "survive". For details on assumptions and results see Forni et al. (2000), Stock and Watson (2002a,b), Bai and Ng (2002).

\subsection{Static method: SW}

Suppose now that for a given $t$ the common components $\chi_{i t}$, for $i \in \mathbb{N}$, span a finite-dimensional vector space $S_{t}$. Stationarity of the common and idiosyncratic components implies that the dimension of $S_{t}$, call it $r$, is independent of $t$ and there exists a "stationary basis" $\mathbf{F}_{t}=\left(F_{1 t} F_{2 t} \cdots F_{r t}\right)^{\prime}$ such that $(2.1)$ can be rewritten in the static form

$$
x_{i t}=\lambda_{i 1} F_{1 t}+\lambda_{i 2} F_{2 t}+\cdots+\lambda_{i r} F_{r t}+\xi_{i t} .
$$


It is easily seen that $r \geq q$, i.e. the number of the so-called static factors $F_{j t}$ is at least equal to the number of dynamic factors, see Forni et al. (2009). A simple example is $x_{i t}=c_{i 0} u_{t}+c_{i 1} u_{t-1}+\cdots+c_{i p} u_{t-p}+\xi_{i t}$, where $q=1, r=p+1$ and a basis for $S_{t}$ is $F_{j t}=u_{t-j+1}, j=1,2, \ldots, p+1$.

Model (2.3) has been predominant in the literature on Dynamic Factor Models, starting with the seminal papers Stock and Watson (2002a,b), Bai and Ng (2002), Forni et al. (2005). The factors $F_{j t}$ and the loadings $\lambda_{i j}$ are estimated using the first $r$ standard principal components. The latter, denoted by $\widehat{\mathbf{F}}_{t}=\left(\begin{array}{llll}\widehat{F}_{1 t} & \widehat{F}_{2 t} & \cdots & \widehat{F}_{r t}\end{array}\right)^{\prime}$, are obtained from the variance-covariance matrix of the observed variables $x_{i t}, i=$ $1,2, \ldots, n, t=1,2, \ldots, T$. Based on the estimated factors, the forecasting equation proposed in Stock and Watson $(2002 \mathrm{a}, \mathrm{b})$, referred to as SW, is the projection of $x_{i, t+h}$ on the space spanned by $\left(\widehat{\mathbf{F}}_{t}, \widehat{\mathbf{F}}_{t-1}, \ldots ; x_{i t}, x_{i, t-1}, \ldots\right)$, where the presence of the terms $x_{i, t-k}$ can be motivated as capturing possible autocorrelation in the idiosyncratic component $\xi_{i t}$ :

$$
\widehat{x}_{i, t+h \mid t}^{S W}=\widehat{\boldsymbol{\alpha}}_{i h}(L) \widehat{\mathbf{F}}_{t}+\widehat{\beta}_{i h}(L) x_{i t}
$$

where $\widehat{\boldsymbol{\alpha}}_{i h}(L)$ is a $1 \times r$ matrix polynomial of degree $g_{i 1, h}$ and $\widehat{\beta}_{i h}(L)$ a scalar polynomial of degree $g_{i 2, h}$.

Estimation of equation (2.4) requires determining three parameters: (i) the num-

ber of static factors $r$, (ii) the degree $g_{i 1, h}$ for $\widehat{\boldsymbol{\alpha}}_{i h}(L)$, (iii) the degree $g_{i 2, h}$ for $\widehat{\beta}_{i h}(L)$. This will be discussed in detail in Section 3.2.1.

\subsection{Dynamic method: FHLZ}

\subsubsection{Infinite dimension of the space $S_{t}$}

A motivation for studying model (2.1) without assumption (2.3), as argued in Forni et al. $(2015,2017)$, is that model $(2.3)$ rules out cases as simple as

$$
x_{i t}=\frac{c_{i}}{1-d_{i} L} u_{t}+\xi_{i t}=c_{i}\left(u_{t}+d_{i} u_{t-1}+d_{i}^{2} u_{t-2}+\cdots\right)+\xi_{i t},
$$


$i \in \mathbb{N}$, where $u_{t}$ is a scalar white noise. If the coefficient $d_{i}$ takes an infinite number of values, for $i \in \mathbb{N}$, then $S_{t}$, the space spanned by the variables $\chi_{i t}=c_{i}\left(u_{t}+d_{i} u_{t-1}+\right.$ $\left.d_{i}^{2} u_{t-2}+\cdots\right), i \in \mathbb{N}$, is infinite-dimensional. This is the case for example if the coefficients $d_{i}$ are drawn from, say, the uniform distribution between -0.8 and 0.8 (with probability one $d_{i}$ takes an infinite number of values) ${ }^{1}$. Infinite dimension of $S_{t}$ obviously occurs in the general model (2.1) if sufficient heterogeneity is allowed for the roots of the polynomials $d_{i f}(L)$.

Forni et al. $(2015,2017)$ construct estimators for the Dynamic Factor Model in its general form (2.1), thus without assuming that $S_{t}$ is finite-dimensional. Such estimators are based on the singularity of the vector $\chi_{t}$. Let us recall that a stochastic vector is singular when it is driven by a number of shocks which is smaller than its dimension, which is the case with $\chi_{t}$ when $n$ is large as compared to $q$. To fix ideas, consider the vector $\chi_{t}^{1}=\left(\begin{array}{llll}\chi_{1 t} & \chi_{2 t} & \cdots & \chi_{q+1, t}\end{array}\right)^{\prime}$, whose dimension is $q+1$, is driven by the $q$-dimensional vector $\mathbf{u}_{t}$ and is therefore (just) singular. Anderson and Deistler (2008) prove that singular VARMA models possess a finite-degree VAR representation for generic values of the parameters. In particular, $\chi_{t}^{1}$, as defined in (2.1) and (2.2), has a representation of the form $\mathbf{A}^{1}(L) \chi_{t}^{1}=\mathbf{R}^{1} \mathbf{u}_{t}$, where $\mathbf{R}^{1}$ is $(q+1) \times q, \mathbf{A}^{1}(L)$ is a $(q+1) \times(q+1)$ finite-degree, stable polynomial matrix, for all the parameters in $(2.2)$, with the exception of a lower-dimensional subset in the parameter space (thus generically). Assuming for simplicity that $n=(q+1) m$ and partitioning $\chi_{t}$ into $(q+1)$-dimensional blocks, we obtain:

$$
\mathbf{A}(L) \chi_{t}=\left(\begin{array}{cccc}
\mathbf{A}^{1}(L) & 0 & \cdots & 0 \\
0 & \mathbf{A}^{2}(L) & \cdots & 0 \\
& & \ddots & \\
0 & 0 & \cdots & \mathbf{A}^{m}(L)
\end{array}\right) \chi_{t}=\mathbf{R u}_{t}=\left(\begin{array}{c}
\mathbf{R}^{1} \\
\mathbf{R}^{2} \\
\vdots \\
\mathbf{R}^{m}
\end{array}\right) \mathbf{u}_{t}
$$

Thus the large-dimensional vector $\chi_{t}$ has a blockwise representation consisting of "small" finite-degree $(q+1)$-dimensional VAR's. Inverting the polynomial matrix

\footnotetext{
${ }^{1}$ If $d_{i}$ can only take a finite number $r$ of values, then the finite-dimension assumption is fulfilled with factors $F_{j t}=\left(1-d_{j} L\right)^{-1} u_{t}, j=1,2, \ldots, r$.
} 
$\mathbf{A}(L)$ in (2.6) (obtained by inversion of the polynomial matrices on its diagonal):

$$
\chi_{t}=[\mathbf{A}(L)]^{-1} \mathbf{R} \mathbf{u}_{t}=\mathbf{B}(L) \mathbf{u}_{t}=\mathbf{B}_{0} \mathbf{u}_{t}+\mathbf{B}_{1} \mathbf{u}_{t-1}+\cdots
$$

Lastly, using $\chi_{t}=\mathbf{x}_{t}-\boldsymbol{\xi}_{t}$, and setting $\mathbf{y}_{t}=\mathbf{A}(L) \mathbf{x}_{t}$ :

$$
\mathbf{y}_{t}=\mathbf{R} \mathbf{u}_{t}+\mathbf{A}(L) \boldsymbol{\xi}_{t}
$$

which is a static factor model for $\mathbf{y}_{t}$.

The estimates $\widehat{\mathbf{A}}^{j}(L), \widehat{\mathbf{R}}^{j}$ and $\widehat{\mathbf{u}}_{t}$ are obtained by the following procedure (see Forni et al. (2017) and Appendix ?? for details):

(i) We estimate the spectral density of $\mathbf{x}_{t}$ by means of a lag-window estimator

$$
\widehat{\boldsymbol{\Sigma}}^{x}(\theta)=\frac{1}{2 \pi} \sum_{k=-T+1}^{T-1} e^{-i k \theta} K\left(\frac{k}{B_{T}}\right) \widehat{\boldsymbol{\Gamma}}_{k}^{x}
$$

where: $\widehat{\Gamma}_{k}^{x}$ is the estimated covariance between $\mathbf{x}_{t}$ and $\mathbf{x}_{t-k}, K$ is a Kernel function, $B_{T}$ is the bandwidth parameter and $2 B_{T}+1$ is the size of the lag window.

(ii) We determine the number of dynamic factors $q$ and obtain from $\widehat{\boldsymbol{\Sigma}}^{x}(\theta)$ an estimate of the spectral density matrix of $\chi_{t}, \widehat{\boldsymbol{\Sigma}}^{\chi}(\theta)$, and of its autocovariance matrices, $\widehat{\boldsymbol{\Gamma}}_{k}^{\chi}$. (iii) The matrices $\widehat{\boldsymbol{\Gamma}}_{k}^{\chi}$ are then used to compute the matrix $\widehat{\mathbf{A}}(L)$, and therefore $\widehat{\mathbf{y}}_{t}=\widehat{\mathbf{A}}(L) \mathbf{x}_{t}$, which is an estimate of the left-hand side of (2.8),

(iv) Lastly, the estimates $\widehat{\mathbf{u}}_{t}$ and $\widehat{\mathbf{R}}$ are obtained by means of the first $q$ standard principal components of $\widehat{\mathbf{y}}_{t}$.

Inverting the matrix $\widehat{\mathbf{A}}(L)$, we obtain the estimated version of (2.7):

$$
\widehat{\chi}_{t}=[\widehat{\mathbf{A}}(L)]^{-1} \widehat{\mathbf{R}} \widehat{\mathbf{u}}_{t}=\widehat{\mathbf{B}}(L) \widehat{\mathbf{u}}_{t}=\widehat{\mathbf{B}}_{0} \widehat{\mathbf{u}}_{t}+\widehat{\mathbf{B}}_{1} \widehat{\mathbf{u}}_{t-1}+\cdots
$$

and the corresponding prediction equation for the common components at horizon $h$ :

$$
\widehat{\chi}_{t+h \mid t}^{F H Z}=\widehat{\mathbf{B}}_{h} \widehat{\mathbf{u}}_{t}+\widehat{\mathbf{B}}_{h+1} \widehat{\mathbf{u}}_{t-1}+\cdots .
$$


Using $\widehat{\boldsymbol{\xi}}_{t}=\mathbf{x}_{t}-\widehat{\chi}_{t}$. Each of the variables $\widehat{\xi}_{i t}$ can be predicted using univariate methods and employed to predict $x_{i t}$ :

$$
\widehat{x}_{i, t+h \mid t}^{F H L Z}=\widehat{\chi}_{i, t+h \mid t}^{F H L Z}+\widehat{\xi}_{i, t+h \mid t}^{F H L Z} .
$$

Estimation of FHLZ requires determining: (i) the number of dynamic factors $q$, (ii) the weights $w_{k}$ of the Kernel function and the lag-window size $2 B+1$ for the estimate $\widehat{\boldsymbol{\Sigma}}^{x}(\theta)$, (iii) the degree of the matrix polynomials $\widehat{\mathbf{A}}^{j}(L)$, (iv) the dynamics of the univariate model for $\widehat{\xi}_{i t}$, see Section 3.2.2 for details.

\subsubsection{Infinite versus finite-dimensional factor space}

No criterion or test has been developed so far about whether the data support finite or infinite dimension for the space $S_{t}$ spanned by the common components. The methods based on finite and infinite dimension of the factor space are studied in the present paper as alternative specifications for the dynamics of the common components, and their relative merits are assessed by their performance in prediction.

As equation (2.6) holds irrespective of whether the space spanned by the variables $\chi_{i t}$ is infinite-dimensional or not, representation (2.6) is more general than (2.3). However, for given $n$ and $T$ a finite-dimensional approximation might be competitive in prediction even if the data were generated by a model with an infinite-dimensional $S_{t}$. For example, in model (2.5) the coefficients $d_{i}$ might be different but all very close to some $d$. In this case, and using (2.4) with $r=1$ could provide better predictions as compared to the correctly specified model. On the other hand, with data generated by (2.3) with a large $r$, the dynamic method could outperform the static method.

In Forni et al. (2017) the static and the dynamic methods have been applied to simulated data in some Monte Carlo experiments. A summary of the results is that: (i) when the data are generated by infinite-dimensional models like (2.5), the estimation of impulse-response functions and predictions obtained by the dynamic method are by far better than those obtained by the static method; (ii) when the data are generated under the finite-dimension assumption, model (2.3), still the dynamic method performs slightly better. In the present paper the comparison between the 
static and dynamic methods is conducted using empirical data, namely the U.S. monthly macroeconomic dataset mentioned in the Introduction and fully described in Section 3.

\subsection{Static, frequency-domain method: FHLR}

As recalled in the Introduction, the method FHLR assumes finite dimension of $S_{t}$ but uses generalized instead of standard principal components. The prediction equation has the same shape as (2.4):

$$
\widehat{x}_{i, t+h \mid t}^{F H L R}=\widehat{\boldsymbol{\alpha}}_{i h}^{G}(L) \widehat{\mathbf{F}}_{t}^{G}+\widehat{\beta}_{i h}^{G}(L) x_{i t},
$$

where $\widehat{F}_{j t}^{G}, j=1,2, \ldots, r$, denotes the $j$-th generalized principal components. Generalized principal components are obtained by the same frequency-domain techniques used in the estimation of FHLZ, see Forni et al. (2005) and Appendix ??.

Estimation of (2.4) requires determining: (i) the number of dynamic factors $q$, the Kernel and the lag-window size for $\widehat{\Sigma}^{x}(\theta)$, like in FHLZ, (ii) the number $r$ of static factors, and the degree of $\widehat{\boldsymbol{\alpha}}_{i h}^{G}(L)$ and $\widehat{\beta}_{i h}^{G}(L)$, like in SW. See Appendix ?? for details.

We refer to SW and FHLR, which are based on representation (2.3), as static methods, and to FHLZ, which is based on (2.6), as a dynamic method. On the other hand, we also refer to FHLR and FHLZ as frequency-domain methods, as both employ the spectral density matrix of the $x$ 's, and to SW as a time-domain method.

\subsection{Alternative Methods: 3PRF, DGR, DMGR}

The three factor models presented above are also compared with three Alternative Methods, two based on factors, one on Bayesian Regression.

3PRF. The Three-Pass Regression Filter, proposed in Kelly and Pruitt (2015), is based on the idea that, given the factors spanning the factor space of the dataset, it is possible to select those who are relevant in the prediction of a given target and discard those which are target-irrelevant. The proposed procedure uses the covariances of 
the variables in the dataset with proxies for the relevant latent factors, the proxies being observable variables either theoretically motivated or automatically selected.

DGR. In a standard finite-dimensional factor model, like in SW or FHLR, Doz et al. (2011) show that the loadings and the factors can be consistently estimated by Quasi-Maximum Likelihood. This estimation method is used here as an alternative to SW.

DMGR. A Bayesian approach to forecasting with a large dataset is proposed in De Mol et al. (2008). The paper studies Bayesian Regression methods under two priors for the coefficients of the variables in the dataset, namely the Gaussian prior and the double-exponential prior. The first prior favors a posterior mode solution in which all variables in the panel have non-zero coefficients, while the second produces a shrinkage of the dataset by selecting a few variables.

\section{Data and Calibration of the Models}

\subsection{Data description, transformations, forecasts}

The dataset consists of 115 U.S. macroeconomic and financial time series observed at monthly frequency between January 1959 and August 2014. To achieve stationarity the series are transformed into first difference of the logarithm (mainly real variables), first difference of yearly difference of the logarithm (prices and wages), first difference (interest rates). A few stationary series are taken in levels, see Appendix ?? for details. No treatment for outliers is applied.

Let $\mathbf{Z}_{\mathbf{t}}=\left(\begin{array}{llll}Z_{1 t} & Z_{2 t} & \cdots & Z_{n t}\end{array}\right)^{\prime}$ be the raw dataset, $\mathbf{X}_{t}=\left(\begin{array}{llll}X_{1 t} & X_{2 t} & \cdots & X_{n t}\end{array}\right)^{\prime}$ the stationary result of the transformations of $\mathbf{Z}_{t}$ just defined. As usual with LargeDimensional Dynamic Factor Models, estimation is carried out using the normalized version of $\mathbf{X}_{t}$ (subtracting the mean and dividing by the standard deviation), here denoted by $\mathbf{x}_{t}=\left(x_{1 t} x_{2 t} \cdots x_{n t}\right)$.

We compute forecasts of $x_{i, t+h}, h=6,12,24$, for the methods SW, FHLZ, FHLR and for a univariate AR. For all four methods we use a rolling ten-year window $[t-119, t]$, and the models are re-estimated for each $t$. 
All the forecasts considered are obtained directly for each horizon $h$, not iterating one-step ahead forecasts, see equation (2.4) for SW, (2.10) for FHLZ and (2.12) for FHLR. Regarding the univariate AR, for each $h$ we estimate $x_{i t}=\gamma_{i h}(L) x_{i, t-h}+v_{i t}$ and use $\widehat{x}_{i, t+h \mid t}=\widehat{\gamma}_{i h}(L) x_{i t}$. The same direct univariate method is used to obtain $\widehat{\xi}_{i, t+h \mid t}^{F H L Z}$ for the idiosyncratic component estimated with FHLZ, so that (2.11) is also a direct forecast.

The forecast of $X_{i, t+h}$ is obtained by restoring the standard deviation and the mean. The forecast at $t$ and horizon $h$ for the method $\mathbf{m}$, with $\mathbf{m}$ ranging over $\mathrm{SW}$, FHLZ, FHLR and AR, is denoted by $\widehat{x}_{i, t+h \mid t}^{\mathrm{m}}$ or $\widehat{X}_{i, t+h \mid t}^{\mathrm{m}}$.

The targets of the final forecasts are usually defined in the literature using our U.S. dataset as the level of the $\log$ of the Industrial Production Index (and of the real variables) and the change, yearly or monthly, of the log of the Consumer Price Index (and of prices and wages), see e.g. Stock and Watson (2002b), D'Agostino and Giannone (2012). As Industrial Production, $\mathrm{IP}_{t}=Z_{1 t}$, is transformed by the first difference of the logarithm, the target at time $t+h$, denoted by $\mathcal{T}_{1, t+h \mid t}$, can be written as $\mathcal{T}_{1, t+h \mid t}=\log \mathrm{IP}_{t+h}=X_{1, t+1}+\cdots+X_{1, t+h}+\log \mathrm{IP}_{t}$, so that

$$
\widehat{\mathcal{T}}_{1, t+h \mid t}^{\mathrm{m}}=\widehat{X}_{1, t+1 \mid t}^{\mathbf{m}}+\cdots+\widehat{X}_{1, t+h \mid t}^{\mathbf{m}}+\log \mathrm{IP}_{t},
$$

and the prediction error, normalized for the horizon's length, is

$$
\mathrm{FE}_{1, t, h}^{\mathrm{m}}=\frac{1}{h}\left(\left(\widehat{X}_{1, t+1 \mid t}^{\mathbf{m}}-X_{1, t+1}\right)+\cdots+\left(\widehat{X}_{1, t+h \mid t}^{\mathbf{m}}-X_{1, t+h}\right)\right) .
$$

For the consumer price index $\mathrm{CPI}_{t}=X_{77, t}$, which is transformed by $(1-L)(1-$ $\left.L^{12}\right) \log$, the target is defined as $\mathcal{T}_{77, t+h \mid t}=\left(1-L^{12}\right) \log \mathrm{CPI}_{t+h}$. Its forecasts are obtained in the same way as $\widehat{\mathcal{T}}_{1, t+h \mid t}^{\mathrm{m}}$, see (3.1). For series that do not require transformation the target is the series itself.

The sample is split into a calibration pre-sample, from February 1960 (some observations at the beginning of the sample are lost due to the difference transformations) to January 1985, and the sample proper, from February 1985 to August 2014. The ten years from February 1975 to January 1985 are used to produce the first forecasts 
within the sample proper. Thus we start by predicting July 1985, January 1986, January 1987 for $h=6,12,24$ respectively. The last forecast is August 2014 for all horizons.

For each predictive model, the forecasting performance is evaluated by its mean square forecast error (MSFE), which is defined as follows:

$$
\mathrm{MSFE}_{i, h}^{\mathbf{m}}=\frac{1}{\left(T_{1}-h\right)-T_{0}+1} \sum_{\tau=T_{0}}^{T_{1}-h}\left[\mathrm{FE}_{i, \tau, h}^{\mathbf{m}}\right]^{2}
$$

where $T_{0}$ and $T_{1}$ denote the first and the last dates either of the pre-sample (calibration) or the sample proper. Replacing the limits of the summation in (3.2) with any time interval within the sample we can measure local forecasting performances.

\subsection{Calibration}

The pre-sample period, February 1960 to January 1985, is used to calibrate the methods SW, FHLZ, FHLR and AR, i.e. to compare the forecasting performance of different specifications for each method. The best specification is then used in the sample for comparison between methods.

To illustrate calibration, consider for example the SW method and let $i=1$, Industrial Production. A crucial parameter is the number $r$ of static factors. We can determine it in different ways. In particular:

$\mathrm{SW}_{1}$. The number $r$ of factors in the static form (2.3) is determined at each time $t$ using the ten-year window $[t-119, t]$, according to Bai and $\mathrm{Ng}^{\prime}$ s criterion $\mathrm{IC}_{2}$, see Bai and $\mathrm{Ng}(2002)^{2}$. No lags are allowed for the factors or the variable to be predicted, thus the prediction equation is (2.4) with $\widehat{\beta}_{i h}(L)=0$ and $\widehat{\boldsymbol{\alpha}}_{i h}(L)$ of degree zero. The model is estimated over the window $[t-119, t]$ and the forecasts $\widehat{\mathcal{T}}_{1, t+h \mid t}^{\mathrm{SW}_{1}}$ computed. As $t+h$ varies from $120+h$ to the end of the pre-sample, we compute a mean square forecast error for each horizon, call it $\mathrm{MSFE}_{1, h}^{\mathrm{SW}_{1}}$.

$\mathrm{SW}_{2}$. The parameter $r$ is kept fixed as the window moves in the pre-sample. Again,

\footnotetext{
${ }^{2}$ We have run some experiments with other criteria, such as Alessi et al. (2010), Onatski (2009), with no significant differences.
} 
no lags for the factors or the variable to be predicted are allowed. With $r$ varying between, say, 3 and 7 we obtain five specifications with corresponding $\mathrm{MSFE}_{1, h}^{\mathrm{SW}_{2, j}}$, $j=3, \ldots, 7$.

Note that different specifications can differ in the value of some parameters: different fixed values of $r$ in $\mathrm{SW}_{2}$, or in the procedure: for example, fixed $r$ as opposed to $r$ determined by the Bai and Ng's criterion. Moreover, each of the six specifications above can be augmented by including lags of the predicted variable and the factors in the prediction equation, see Section 3.2.1.

To compare specifications $\mathbf{m}_{1}$ and $\mathbf{m}_{2}$ of method $\mathbf{m}$ at horizon $h=6,12,24$ for the variable $i$, we use the ratio

$$
\operatorname{RMSFE}_{i, h}^{\mathbf{m}_{1} / \mathbf{m}_{2}}=\frac{\operatorname{MSFE}_{i, h}^{\mathbf{m}_{1}}}{\operatorname{MSFE}_{i, h}^{\mathbf{m}_{2}}}
$$

Because in many cases no specification prevails uniformly across different horizons, we choose according to the average of the ratio (3.3) over the three horizons. The calibration procedure is limited to aggregate industrial production, $\mathrm{IP}_{t}=Z_{1, t}$, and

consumer price, $\mathrm{CPI}_{t}=Z_{77, t}$. The chosen specifications are then used, respectively, in the forecast of disaggregated real and nominal variables.

\subsubsection{Calibration of SW}

It is easily seen that detailed consideration of all the alternatives leads to a large number of specifications:

(i) Firstly, to determine $r$ we can choose between $\mathrm{SW}_{1}$, with different possible criteria, or $\mathrm{SW}_{2}$, with $r$ independent of $t$, to be chosen in an interval of values.

(ii) Each of the alternatives in (i) should be combined with the alternatives in the determination of the degree of the polynomial $\widehat{\beta}_{i h}(L)$, i.e. the criterion, AIC or BIC in particular, and the maximum lag used in the criterion.

(iii) Same as in (ii) for the lags of the factors, i.e. the vector polynomial $\widehat{\boldsymbol{\alpha}}_{i h}(L)$. Again, there are alternative criteria and maximum lags.

(iv) Same as in (ii) with lags for both the factors and the predicted variable. 
(v) The polynomial degrees in (ii), (iii) and (iv) can be kept fixed as $t$ moves and be chosen within intervals of values.

An exploration of the "cartesian product" of the alternatives outlined above with elementary methods is impossible. We limit ourselves to a recursive scheme. Firstly, we choose the method to determine $r$ by running $\mathrm{SW}_{1}$ and $\mathrm{SW}_{2}$, thus without lags of the target or the factors. Then, we augment the selected specifications with lags of the target, the factors, or both.

S1. For $i=1$ (IP) and $i=77$ (CPI), $h=6,12,24$, we compute the ratios RMSFE $\mathbf{m}_{i, h}^{\mathbf{m}_{1} / \mathbf{m}_{2}}$ where: (1) $\mathbf{m}_{2}$ is $\mathrm{SW}_{2}$ with $r$ equal to $5,(2) \mathbf{m}_{1}$ is either $\mathrm{SW}_{1}$ or $\mathrm{SW}_{2}$ with $r=1, \ldots, 8$. The results are reported in Table ??, Panel SW:S1. We see that the best models are: (I) $\mathrm{SW}_{2}$ with $r=6$ for IP with $r=7,8$ very close, (II) $\mathrm{SW}_{2}$ with $r=5$ for CPI, the second best being $\mathrm{SW}_{1}$. The two best models are denoted by $\mathrm{SW}_{2}(6)$ and $\mathrm{SW}_{2}(5)$ respectively.

S2. We run the prediction equation (2.4) with $r=6, r=5$ for IP and CPI respectively, augmented with lags for the predicted variable. The degree of $\widehat{\beta}_{i h}(L)$ is determined by the AIC or the BIC criteria setting the maximum number of lags to 15. The results are reported in Appendix ??, Panel SW:S2 of Table ??, the benchmark for the RMSFE being $\mathrm{SW}_{2}(6)$ for IP and $\mathrm{SW}_{2}(5)$ for CPI. For both IP and $\mathrm{CPI}$ the best result is obtained using the BIC criterion. On average they are worse though not far from $\mathrm{SW}_{2}(6)$ and $\mathrm{SW}_{2}(5)$ respectively.

S3 and $S_{4}$. The models $\mathrm{SW}_{2}(6)$ and $\mathrm{SW}_{2}(5)$ augmented with lags of the factors are run. The degree of $\widehat{\boldsymbol{\alpha}}_{i h}(L)$ is determined by the AIC and the BIC criteria setting the maximum number of lags to 15. Again, the results are worse as compared to $\mathrm{SW}_{2}(6)$ and $\mathrm{SW}_{2}(5)$. Lastly, $\mathrm{SW}_{2}(6)$ and $\mathrm{SW}_{2}(5)$ are augmented with both lags of the factors and of the predicted variable. The results are very poor, see Appendix ??, Table ??, Panels SW:S3,S4.

In conclusion, our exploration of the space of possible SW specifications points to $\mathrm{SW}_{2}(6)$ and $\mathrm{SW}_{2}(5)$ as good models for IP and CPI respectively. They are our first choice for SW in the in-sample comparison.

Note that the same variables $x_{i t}$ (stationary and normalized) are used to estimate both the four models and the "original forecasts" $\widehat{x}_{i, t+h \mid t}^{\mathbf{m}}$. The final forecasts $\widehat{\mathcal{T}}_{1, t+h \mid t}^{\mathrm{m}}$ 
are obtained from $\widehat{x}_{i, t+h \mid t}^{\mathbf{m}}$ by restoring mean and standard deviation, and cumulating if necessary. The same method is used in D'Agostino and Giannone (2012). An alternative method is used in Stock and Watson (2002a). For example, the forecast of $\log \mathrm{IP}_{t+h}$ is obtained by projecting $\log \mathrm{IP}_{t+h}-\log \mathrm{IP}_{t}$ on the factors $\mathbf{F}_{t}$ (and lags) and $\log \mathrm{IP}_{t}-\log \mathrm{IP}_{t-1}$ (and lags), thus without using the cumulation in equation (3.1). Experiments with the alternative method for SW did not produce significant differences in $\mathrm{MSFE}_{i, h}^{\mathrm{SW}}$, at all horizons, both in the pre-sample and the sample proper.

Lastly, let us remark that in our calibration we are not considering forecast combinations based on the "basic" specifications considered and compared above. For example, we might average over the forecasts obtained by using different numbers of factors or lags. Moreover, we might experiment with optimal combination weights as opposed to simple averaging, see e.g. Timmermann (2006) for a review. However, the results of some random attempts with forecasts combinations were not encouraging. On the other hand, calibration of SW and the other methods with basic specifications is already fairly heavy, thus we decided to leave systematic exploration of this possible improvement, for SW and the other methods, to future research.

\subsubsection{Calibration of FHLZ, FHLR and AR}

S1, ordering of the variables. FHLZ is based on equations (2.9) and (2.10), which are obtained from inversion of the estimated version of (2.6). Now, a change in the order of the variables $x_{i t}$ and $\chi_{i t}$ obviously causes a change in the matrices $\mathbf{A}^{j}(L)$ and $\mathbf{R}^{j}$ in (2.6). However, under mild assumptions, see Forni et al. (2017), no change occurs in the (infinite) moving average polynomials in (2.7). For example, the $q$ moving average polynomials of $\chi_{1 t}$ in (2.7), loading $u_{f t}, f=1,2, \ldots, q$, do not change if the variables $\chi_{i t}, i=2, \ldots, q+1$ are replaced by other variables in the first block of size $q+1$.

Things change when $\mathbf{A}^{j}(L)$ and $\mathbf{R}^{j}$ are replaced by their estimated counterparts $\widehat{\mathbf{A}}^{j}(L)$ and $\widehat{\mathbf{R}}^{j}$. Because the idiosyncratic components have not yet been completely erased and because their size is heterogeneous, each of the estimated polynomials in (2.10) depends on the grouping of the variables $x_{i t}$ and therefore on the ordering of 
the variables $x_{i t}$ in the dataset.

Considering for example $x_{1 t}$, we have a large number of predictors, one for each grouping of the variables $x_{i t}$ into subvectors of dimension $q+1$. Of course, choosing the grouping corresponding to the order in which the dataset is delivered is arbitrary. On the other hand, as we argue in Forni et al. (2017), averaging over the forecasts of $x_{1 t}$ corresponding to all possible groupings we would obtain a forecast that: (1) depends only on the variables in the dataset irrespective of their order, (2) has an expected performance that is not worse as compared to that provided by any single grouping.

Of course such an average is unfeasible for $n$ large. Fortunately, as we show in Appendix ??, averaging over $N_{\text {per }}=100$ random permutations of the variables $x_{i t}$ we obtain (2) and a very good approximation to (1).

The remaining steps of the calibration of FHLZ determine the bandwidth parameter $B$ and the degree of the $(q+1)$-dimensional VAR's. As the procedure goes much in the same way as in the calibration of SW, the details are given in Appendix ??. The resulting specification uses the triangular Kernel, $B=30, q$ is determined at each $t$ by the Hallin-Liška criterion, the degree of the VAR's is determined by the AIC criterion with maximum lag 5.

Details on the calibration of FHLR can also be found in Appendix ??. The selected specification uses the Triangular Kernel with $B=40, q$ is chosen at each $t$ with the Hallin-Liška criterion, $r$ is fixed and equal to 6 and 5 for IP and CPI respectively, the degree of $\widehat{\boldsymbol{\alpha}}_{i h}^{G}(L)$ is zero and $\widehat{\beta}_{i h}^{G}(L)=0$.

Regarding AR, we determine the number of lags at each $t$, for each $h$, by the BIC criterion with maximum lag 13 . This is the best among several specifications both in the pre-sample and the sample proper.

\subsubsection{Calibration of 3PRF, DGR and DMGR}

3PRF. We run the 3PRF in the automatic-proxies version, see Kelly and Pruitt (2015), p. 299. Comparing the results, for IP and CPI, with different numbers of proxies, in the pre-sample period we find values between 1 and 2 , depending on 
the variable and the horizon. However, in the sample proper, the best results are obtained with just one proxy.

DGR. Calibration in the pre-sample period points to a fixed number of static factors, with $r=2$ or 3 for IP, $r=4$ or 5 for CPI (depending on the horizon). The best results in the sample proper are obtained with $r=2$ for IP and $r=4$ for CPI.

DMGR. The model is calibrated in the pre-sample period by choosing the in-sample residual variance corresponding to the best forecasting performance (see Table 2 in De Mol et al. (2008)). The selected in-sample residual variance is: (i) 0.3 for IP, all horizons, and CPI, $h=6$ and $h=12$, (ii) 0.4 for CPI, $\mathrm{h}=24$.

\section{Results}

\subsection{Industrial Production and Inflation}

We now compare the performance of the factor models in the prediction of IP and CPI over the sample starting in February 1985. For FHLZ and FHLR we stick to the specifications selected in the previous section. For SW we ran in the sample several of the specifications that were discarded in the pre-sample. None of them outperforms $\mathrm{SW}_{2}(5)$ for CPI. However, $\mathrm{SW}_{2}(5)$ outperforms $\mathrm{SW}_{2}(6)$ for IP, the latter having been selected in the calibration. We report the results obtained with both $\mathrm{SW}_{2}(5)$ and $\mathrm{SW}_{2}(6)$ for IP. However, when commenting on $\mathrm{SW}$ we always refer to $\mathrm{SW}_{2}(5)$, i.e. the specification performing better in the sample proper.

In Table 1 we report the average performance, measured by the RMSFE, of the three factor models (and the Alternative Methods) relative to AR. We give results for the Great Moderation, or pre-crisis period, starting with February 1985 and ending at December 2007, the beginning of the Great Recession (from December 2007 to June 2009), Panel A, and the full sample period, from February 1985 to September 2014, Panel B.

There are two strong reasons for splitting the sample. Firstly, IP, CPI and the whole dataset exhibit marked instability during the Great Recession. Regarding IP and CPI, this is clearly visible in the plot of the targets $(1-L) \log (\mathrm{IP})$ and 
$(1-L)\left(1-L^{12}\right) \log (\mathrm{CPI})$, see Figure 1, top graphs. We observe a marked change in the mean of the first in the Great Recession. The second is stable in the mean, though exhibiting two outliers. Regarding the whole dataset, a marked change in its covariance structure is roughly but convincingly illustrated in the lower graph of Figure 1, where we plot the sum of squares $\sum_{\tau=T_{0}}^{t} \sum_{i=1}^{115} x_{i \tau}^{2}$, for $t$ running from February 1985 to August 2014, the sample proper (recall that $x_{i \tau}$ is the dataset after transformation and normalization, see Section 3.1). We observe a steady growth with a particularly sharp increase in the slope during the Great Recession.

Secondly, as a consequence of that instability, the relative forecasting performance of the factor models and the AR changes dramatically during the Great Recession. This is clearly illustrated in Figures 2 and 3. The solid line is the graph of the difference between the Square Forecast Error with methods $\mathbf{m}_{1}$ and $\mathbf{m}_{2}$, FHLZ and SW for example, relative to IP and CPI, at each horizon, normalized by its estimated standard deviation and smoothed by a centered moving average of length $M=$ 61, with the coefficients equal to $1 / M$. Giacomini and Rossi (2010) use it to test against the null of equal local performance of two forecasting methods. The zero horizontal line indicates equal performance, the dotted lines indicate the 5-percent critical values, so that $\mathbf{m}_{1}$ outperforms (underperforms) $\mathbf{m}_{2}$ locally, at the 5-percent significance level, when the solid line is below (above) the lower (upper) dashed line. Because the moving averages are of length 61 and centered, the first and last 30 values are not computed or graphed.

In addition to testing for local equal performance, limiting the sample to the relatively stable pre-crisis period, we use the Diebold-Mariano test (see Diebold and Mariano (1995)) against the null of global equal performance of two predictors. Our main results are:

IP. FHLZ outperforms the other three methods in the pre-crisis period on average over the three horizons. It is slightly outperformed by FHLR at horizon 24, see Panel A in Table 1. The null of equal performance with FHLZ, in the pre-crisis period, is rejected for AR (at the 1\% significance level), SW (at 5\% for horizon 6 and 12, 10\% for horizon 24). It is also rejected at the $10 \%$ level 
for FHLR for horizons 6 and 12. All the $p$-values are reported in Table 2. The performance of FHLZ is somewhat improving before the crisis with respect to the three other methods at horizons 12 and 24, see Figure 2. During the crisis, see again Figure 2, SW and FHLR behave significantly (Giacomini-Rossi test) better than FHLZ and AR, while AR performs significantly better than FHLZ. However, with the end of the crisis almost all the solid lines are clearly heading back to the pre-crisis pattern. On average over the whole sample, FHLZ is outperformed by FHLR and SW at horizons 6 and 12. All methods do better than AR with the exception of SW at horizon 24, see Panel B in Table 1.

CPI. FHLZ outperforms the other three methods in the pre-crisis period on average over the three horizons. It is however outperformed at horizon 24 by FHLR and, slightly, SW, see Panel A in Table 1. The null of equal performance with FHLZ, in the pre-crisis period, is rejected for AR (at levels $10 \%, 5 \%$ and $1 \%$ ), SW (at $10 \%$ for horizon 6 and 12). It is also rejected at the $10 \%$ level for FHLR for horizon 6 , see Table 2. In this case the crisis has a negative effect on the performance of all three factor methods as compared to AR, see Figure 3. However, on average over the full sample, the best method remains FHLZ, with the exception of horizon 24, for which it is outperformed by SW. In many cases, though not as regularly as for IP, with the end of the crisis the solid lines in Figure 3 go back to the pre-crisis pattern.

To understand our results let us recall that the factor models employed here are based on the assumption of stationarity and co-stationarity (after suitable transformations) of the variables in the dataset, while the AR method only requires stationarity of the variable to be predicted. During the Great Moderation, when such assumptions are by and large fulfilled, the relative performance of the factor models and the AR change little, see again the pre-crisis period in Figures 2 and 3. In particular, FHLZ outperforms the other methods, consistently with the results obtained with simulated stationary data in Forni et al. (2017). On the other hand, as soon as the crisis breaks out, as already observed,

(I) The targets $(1-L) \log (\mathrm{IP})$ and $(1-L)\left(1-L^{12}\right) \log (\mathrm{CPI})$ exhibit unstable 
behavior, see again Figure 1, top graphs. Both the factor and the AR models are affected.

(II) The autocovariance structure of the dataset changes abruptly, see again Figure 1, lower graph. This instability, affecting only the factor models, causes a deterioration in the estimation of the factors and of the loadings, i.e. the coefficients in (2.4), (2.10), (2.12).

In the case of CPI, where instability (I) is mild, all factor models loose ground with respect to the AR model. FHLZ remains the best method in the full sample, though by little with respect to AR. Moreover, the performance of the factor models relative to one another does not change much.

In the case of IP, where the instability (I) is much more important. The AR model, which is only based on the target, is strongly affected and its performance looses ground with respect to FHLR and SW. On the other hand, FHLZ, which is more dependent on the stationarity assumptions than the other factor models, looses ground with respect to all other methods.

The results for 3PRF, DGR and DMGR, see Sections 2.4 and 3.2.3, are reported in Table 1, last three columns.

IP. In the pre-crisis period $3 \mathrm{PRF}$ and DGR are very close on average to FHLR and therefore outperform SW but are outperformed by FHLZ. DMGR is outperformed by AR. In the crisis period all the Alternative Methods perform poorly, so that none of them outperforms on average AR in the full sample.

CPI. In the pre-crisis period 3PRF performs on average like FHLZ, this being the result of a very good performance at horizon 24. DMGR and DGR perform like SW. In the full-sample period DMGR performs extremely well at horizon 24 and, in spite of the bad performance at horizon 6, slightly outperforms FHLZ on average. ${ }^{3}$

\footnotetext{
${ }^{3}$ Our result with DMGR on CPI, a good performance during the crisis as opposed to the greatmoderation period, is consistent with Table 2, p. 323 in De Mol et al. (2008), where the performance is very good for the period 1971-1984 but poor for 1985-2002.
} 
Summing up, FHLZ is confirmed as the best method in the pre-crisis period both for IP and CPI, FHLR as the best for IP in the full sample, while DMGR has the best performance for CPI in the full sample.

\subsection{Forecasting the whole dataset}

We now extend the pseudo real-time comparison of our four methods to the whole dataset. For some of the variables the AR method outperforms the factor models. Precisely, we find that for 23 variables the AR outperforms by at least 10 percent all the factor models for at least one prediction horizon. This subset of variables, which includes housing, category 4 in Table ??, Appendix ??, is excluded. ${ }^{4}$

For real variables we use the specifications adopted for IP (we only ran $\mathrm{SW}_{2}(5)$ ), while for the nominal variables those adopted for CPI. For every group of variables, in Table 3 we report the mean RMSE within the group. The best performance is given in bold. We see that FHLZ and FHLR generally perform better than SW, the latter being the most accurate only for employment 6 and 12 steps ahead. All in all, FHLR is more accurate for the real variables, i.e. IP, Employment, Unemployment Rate, Inventories, while FHLZ is more accurate for nominal variables, i.e. Prices, Wages, Interest Rates, Money and Stock Prices. For Exchange Rates and Wages at horizon 12 the AR is still the most accurate model. Considering median values rather than means we obtain similar results.

In Table 4 the distribution of the RMSE of the models is calculated excluding the same variables as before. Only FHLZ improves at every horizon upon AR for more than the half of the series. FHLR does so only 24-step ahead, is less accurate than AR 6-step ahead by 4.1 percent and 2.1 percent 12-step ahead. Furthermore, FHLZ is roughly as accurate as AR even at his 75 -th percentile. SW is outperformed by frequency domain models at most percentiles and horizons. Its performance deteriorates as the predictive horizon increases while the contrary holds for FHLR and FHLZ. Among the frequency domain methods FHLZ performs better at the

\footnotetext{
${ }^{4}$ This result holds for our monthly dataset. In other works, with quarterly datasets, dynamic factor models are successfully applied to housing market data, see Luciani (2015), Stock and Watson (2008) and Moench and Ng (2011).
} 
95-th percentile and FHLR is more accurate at the 5-th percentile.

\section{Conclusions}

The paper has compared the forecasting performance of FHLZ, FHLR, SW and AR for a U.S. dataset including the Great Moderation, the Great Recession and the subsequent recovery.

We find that during the Great Moderation, when the dataset is relatively stable, FHLZ significantly prevails for both IP and CPI.

Over the full sample, the performance of FHLZ remains the best for CPI, though all factor models loose ground with respect to the simple AR model. FHLR and SW, in this order, become the best models for IP, thus exhibiting more robustness than FHLZ in a situation where both the target variable and the whole dataset undergo instability.

Forecasting each single series in the U.S. dataset for the full sample confirms the above results, with FHLZ being the best method for the nominal variables, FHLR for the real variables.

A robustness check of the results obtained with the U.S. data has been conducted on a European dataset consisting of 176 macroeconomic and financial time series for the Euro Area, observed at monthly frequency between January 1985 and August 2016, see Appendix ?? for details. The performance of the three factor models, relative to one another, is confirmed in the pre-crisis period. In the full sample FHLR is the best method, albeit slightly, for both IP (as with the U.S. dataset) and CPI. However, with respect to the U.S. dataset, an important difference is the bad performance of all the factor models relative to AR for CPI in the pre-crisis period. See the results in Appendix ??.

Based on the data, U.S. and European, and the models employed in the present paper: (a) In stable periods FHLZ can be strongly recommended for IP and CPI; (b) when the data include unstable subperiods, such as the Great Recession, FHLR can be recommended for IP, FHLZ and FHLR for CPI.

Instability is of course a major issue in forecasting. Its impact on factor estimation 
and factor-based forecasts has been studied and discussed in Stock and Watson (2002a), D'Agostino et al. (2007), Banerjee et al. (2008), Stock and Watson (2009), D'Agostino et al. (2013), Clements (2015). The present paper however is the first to consider the effect of the Great Recession and the subsequent recovery in a pseudo real-time forecasting exercise using factor models. ${ }^{5}$

\footnotetext{
${ }^{5}$ More recent research has focused on (i) detecting different forms of instability in factor models, see e.g. Breitung and Eickmeier (2011), Han and Inoue (2014), Yamamoto and Tanaka (2015), Chen et al. (2014), Barigozzi et al. (2016), (ii) consistent factor estimation under breaks, Bates et al. (2013), Ma and Su (2016), Cheng et al. (2016), Massacci (2016), (iii) modeling time varying factor models Del Negro and Otrok (2008), Mikkelsen et al. (2015). However, in our knowledge, no attempt has been made so far to use these models in a pseudo real-time forecasting exercise.
} 


\section{References}

Albano, D. (2016). Latent factors in large-dimensional datasets: forecasting and data analysis using factor models. PhD thesis, Università degli Studi di Napoli Federico II.

Alessi, L., Barigozzi, M., and Capasso, M. (2010). Improved penalization for determining the number of factors in approximate factor models. Statistics \& Probability Letters, 80(23-24):1806-1813.

Amengual, D. and Watson, M. W. (2007). Consistent estimation of the number of dynamic factors in a large $\mathrm{N}$ and $\mathrm{T}$ panel. Journal of Business \& Economic Statistics, 25(1):91-96.

Anderson, B. and Deistler, M. (2008). Properties of zero-free transfer function matrices. SICE Journal of Control, Measurement and System Integration, 1(4):284-92.

Bai, J. and Ng, S. (2002). Determining the number of factors in approximate factor models. Econometrica, 70(1):191-221.

Banerjee, A., Marcellino, M., and Masten, I. (2008). Forecasting macroeconomic variables using Diffusion Indexes in short samples with structural change. In Forecasting in the presence of structural breaks and model uncertainty, volume 3 of Frontiers of Economics and Globalization, pages 149-194. Emerald Group Publishing.

Barigozzi, M., Cho, H., and Fryzlewicz, P. (2016). Simultaneous multiple changepoint and factor analysis for high-dimensional time series. arXiv:1612.06928 [stat].

Bates, B. J., Plagborg-Möller, M., Stock, J. H., and Watson, M. W. (2013). Consistent factor estimation in dynamic factor models with structural instability. Journal of Econometrics, 177(2):289-304.

Boivin, J. and Ng, S. (2005). Understanding and comparing factor-based forecasts. International Journal of Central Banking, 1(3). 
Breitung, J. and Eickmeier, S. (2011). Testing for structural breaks in dynamic factor models. Journal of Econometrics, 163(1):71-84.

Chen, L., Dolado, J., and Gonzalo, J. (2014). Detecting big structural breaks in large factor models. Journal of Econometrics, 180(1):30-48.

Cheng, X., Liao, Z., and Schorfheide, F. (2016). Shrinkage estimation of highdimensional factor models with structural instabilities. The Review of Economic Studies, 83(4):1511-1543.

Clements, M. P. (2015). Real-time factor model forecasting and the effects of instability. Computational Statistics \& Data Analysis. to appear.

D'Agostino, A., Gambetti, L., and Giannone, D. (2013). Macroeconomic forecasting and structural change. Journal of Applied Econometrics, 28(1):82-101.

D'Agostino, A. and Giannone, D. (2012). Comparing alternative predictors based on large-panel factor models. Oxford Bulletin of Economics and Statistics, 74(2):306326.

D’Agostino, A., Giannone, D., and Surico, P. (2007). (Un)Predictability and macroeconomic stability. CEPR Discussion Paper 6594, C.E.P.R. Discussion Papers.

De Mol, C., Giannone, D., and Reichlin, L. (2008). Forecasting using a large number of predictors: Is bayesian shrinkage a valid alternative to principal components? Journal of Econometrics, 146(2):318-328.

Del Negro, M. and Otrok, C. (2008). Dynamic factor models with time-varying parameters: measuring changes in international business cycles. SSRN Scholarly Paper ID 1136163, Social Science Research Network, Rochester, NY.

den Reijer, A. H. J. (2005). Forecasting Dutch GDP using large scale factor models. DNB Working Paper 2005/28, De Nederlandsche Bank.

Diebold, F. X. and Mariano, R. S. (1995). Comparing predictive accuracy. Journal of Business $\&$ Economic Statistics, 13(3):253-263. 
Doz, C., Giannone, D., and Reichlin, L. (2011). A Quasi-Maximum Likelihood approach for large, approximate Dynamic factor models. Review of Economics and Statistics, 94(4):1014-1024.

Eickmeier, S. and Ziegler, C. (2008). How successful are dynamic factor models at forecasting output and inflation? A meta-analytic approach. Journal of Forecasting, 27(3):237-265.

Forni, M., Giannone, D., Lippi, M., and Reichlin, L. (2009). Opening the black box: structural factor models with large cross sections. Econometric Theory, 25(05):1319-1347.

Forni, M., Hallin, M., Lippi, M., and Reichlin, L. (2000). The generalized dynamicfactor model: Identification and estimation. Review of Economics and Statistics, $82(4): 540-554$.

Forni, M., Hallin, M., Lippi, M., and Reichlin, L. (2005). The generalized dynamic factor model: One-sided estimation and forecasting. Journal of the American Statistical Association, 100:830-840.

Forni, M., Hallin, M., Lippi, M., and Zaffaroni, P. (2015). Dynamic factor model with infinite dimensional factor space: Representation. Journal of Econometrics, 185:359-371.

Forni, M., Hallin, M., Lippi, M., and Zaffaroni, P. (2017). Dynamic factor models with infinite-dimensional factor spaces: Asymptotic analysis. Journal of Econometrics, 199(1):72-92.

Giacomini, R. and Rossi, B. (2010). Forecast comparisons in unstable environments. Journal of Applied Econometrics, 25(4):595-620.

Hallin, M. and Liška, R. (2007). Determining the number of factors in the general dynamic factor model. Journal of the American Statistical Association, 102(478):603617. 
Han, X. and Inoue, A. (2014). Tests for parameter instability in dynamic factor models. Econometric Theory, pages 1-36.

Kapetanios, G. and Marcellino, M. (2009). A parametric estimation method for dynamic factor models of large dimensions. Journal of Time Series Analysis, 30:208-238.

Kelly, B. and Pruitt, S. (2015). The three-pass regression filter: A new approach to forecasting using many predictors. Journal of Econometrics, 186(2):294-316.

Luciani, M. (2015). Monetary policy and the housing market: A structural factor analysis. Journal of Applied Econometrics, 30(2):199-218.

Ma, S. and Su, L. (2016). Estimation of large dimensional factor models with an unknown number of breaks. Working Paper 05-2016, Singapore Management University, School of Economics.

Massacci, D. (2016). Unstable diffusion indexes: With an application to Bond Risk Premia. SSRN Scholarly Paper ID 2816534, Social Science Research Network, Rochester, NY.

McCracken, M. W. and Ng, S. (2016). FRED-MD: A Monthly Database for macroeconomic research. Journal of Business \& Economic Statistics, 34(4):574-589.

Mikkelsen, J. G., Hillebrand, E., and Urga, G. (2015). Maximum Likelihood Estimation of time-varying loadings in high-dimensional factor models. CREATES Research Paper 2015-61, Department of Economics and Business Economics, Aarhus University.

Moench, E. and Ng, S. (2011). A hierarchical factor analysis of U.S. housing market dynamics. The Econometrics Journal, 14(1):C1-C24.

Onatski, A. (2009). Testing hypotheses about the number of factors in large factor models. Econometrica, 77(5):1447-1479. 
Peña, D. and Poncela, P. (2004). Forecasting with nonstationary dynamic factor models. Journal of Econometrics, 119(2):291-321.

Schumacher, C. (2007). Forecasting German GDP using alternative factor models based on large datasets. Journal of Forecasting, 26(4):271-302.

Stock, J. and Watson, M. (2009). Forecasting in dynamic factor models subject to structural instability. The Methodology and Practice of Econometrics. A Festschrift in Honour of David F. Hendry, 173:1-57.

Stock, J. H. and Watson, M. W. (2002a). Forecasting using principal components from a large number of predictors. Journal of the American Statistical Association, 97(460):1167-1179.

Stock, J. H. and Watson, M. W. (2002b). Macroeconomic forecasting using diffusion indexes. Journal of Business \& Economic Statistics, 20(2):147-162.

Stock, J. H. and Watson, M. W. (2005). Implications of dynamic factor models for VAR analysis. NBER Working Paper 11467, NBER.

Stock, J. H. and Watson, M. W. (2008). The evolution of national and regional factors in US housing construction. Princeton University.

Stock, J. H. and Watson, M. W. (2012). Generalized shrinkage methods for forecasting using many predictors. Journal of Business 83 Economic Statistics, 30(4):481493.

Timmermann, A. (2006). Forecast Combinations, volume 1 of Handbook of Economic Forecasting, chapter 4, pages 135-196. Elsevier.

Yamamoto, Y. and Tanaka, S. (2015). Testing for factor loading structural change under common breaks. Journal of Econometrics, 189(1):187-206. 
Table 1: Mean Square Forecast Error Relative to AR for U.S. data

PANEL A: Pre Crisis (1985 : 1 - $2007: 11)$

\begin{tabular}{|c|c|c|c|c|c|c|c|}
\hline \multicolumn{8}{|c|}{ IP } \\
\hline & FHLZ & FHLR & $\mathrm{SW}_{2}(5)$ & $\mathrm{SW}_{2}(6)$ & 3PRF1 & DMGR & DGR2 \\
\hline$h=6$ & 0.85 & 0.97 & 1.05 & 1.06 & 0.95 & 0.97 & 0.95 \\
\hline$h=12$ & 0.90 & 0.99 & 1.10 & 1.19 & 0.99 & 1.05 & 0.97 \\
\hline$h=24$ & 0.97 & 0.96 & 1.14 & 1.32 & 1.02 & 1.31 & 1.04 \\
\hline mean & 0.90 & 0.98 & 1.10 & 1.19 & 0.99 & 1.11 & 0.99 \\
\hline \multicolumn{8}{|c|}{ CPI } \\
\hline & FHLZ & FHLR & $\mathrm{SW}_{2}(5)$ & 3PRF1 & DMGR & DGR4 & \\
\hline$h=6$ & 0.92 & 1.02 & 1.04 & 0.96 & 1.01 & 1.01 & \\
\hline$h=12$ & 0.84 & 0.94 & 1.02 & 0.89 & 0.92 & 0.99 & \\
\hline$h=24$ & 0.86 & 0.82 & 0.85 & 0.78 & 0.96 & 0.88 & \\
\hline mean & 0.87 & 0.93 & 0.97 & 0.88 & 0.96 & 0.96 & \\
\hline
\end{tabular}

PANEL B: Full Sample (1985 : 1 - $2014: 8)$

\begin{tabular}{|c|c|c|c|c|c|c|c|}
\hline \multicolumn{8}{|c|}{ IP } \\
\hline & FHLZ & FHLR & $\mathrm{SW}_{2}(5)$ & $\mathrm{SW}_{2}(6)$ & 3PRF1 & DMGR & DGR2 \\
\hline$h=6$ & 0.95 & 0.87 & 0.86 & 0.90 & 1.09 & 0.97 & 1.14 \\
\hline$h=12$ & 0.94 & 0.88 & 0.86 & 0.98 & 0.99 & 1.09 & 1.11 \\
\hline$h=24$ & 0.97 & 0.93 & 1.06 & 1.17 & 0.97 & 1.09 & 1.03 \\
\hline mean & 0.95 & 0.89 & 0.93 & 1.02 & 1.02 & 1.05 & 1.09 \\
\hline \multicolumn{8}{|c|}{ CPI } \\
\hline & FHLZ & FHLR & $\mathrm{SW}_{2}(5)$ & 3PRF1 & DMGR & DGR4 & \\
\hline$h=6$ & 0.95 & 1.09 & 1.14 & 0.99 & 1.04 & 1.19 & \\
\hline$h=12$ & 0.98 & 1.15 & 1.06 & 1.00 & 0.98 & 1.17 & \\
\hline$h=24$ & 1.04 & 1.01 & 0.95 & 1.02 & 0.90 & 1.10 & \\
\hline mean & 0.99 & 1.08 & 1.05 & 1.00 & 0.97 & 1.15 & \\
\hline
\end{tabular}

Notes. Mean Square Forecast Error (MSFE), relative to AR, for IP and CPI, in the Pre-Crisis and the Full-Sample periods. For IP we display both the results of $\mathrm{SW}_{2}(6)$, the method selected in the calibration sample, and $\mathrm{SW}_{2}(5)$. The methods compared are the three factor models and the three Alternative Methods. The best result for each horizon, over all methods, in bold. 
Table 2: Diebold-Mariano test for the pre-crisis period: $p$-values

\begin{tabular}{|c|c|c|c|c|c|c|c|}
\hline \multicolumn{8}{|c|}{ IP } \\
\hline & FHLZ vs $\mathrm{SW}_{2}(5)$ & FHLR vs $\mathrm{SW}_{2}(5)$ & FHLZ vs FHLR & FHLZ vs AR & FHLR vs AR & $\mathrm{SW}_{2}(5)$ vs $\mathrm{AR}$ & $\mathrm{SW}_{2}(6)$ vs $\mathrm{AR}$ \\
\hline $\mathrm{h}=6$ & 0.05 & 0.03 & 0.10 & 0.00 & 0.44 & 0.68 & 0.72 \\
\hline $\mathrm{h}=12$ & 0.05 & 0.03 & 0.11 & 0.00 & 0.48 & 0.78 & 0.94 \\
\hline $\mathrm{h}=24$ & 0.07 & 0.04 & 0.56 & 0.09 & 0.25 & 0.91 & 0.98 \\
\hline \multicolumn{8}{|c|}{ CPI } \\
\hline & FHLZ vs SW & FHLR vs SW & FHLZ vs FHLR & FHLZ vs AR & FHLR vs AR & $\mathrm{SW}$ vs $\mathrm{AR}$ & \\
\hline $\mathrm{h}=6$ & 0.07 & 0.22 & 0.08 & 0.09 & 0.53 & 0.58 & \\
\hline $\mathrm{h}=12$ & 0.07 & 0.02 & 0.15 & 0.02 & 0.31 & 0.53 & \\
\hline $\mathrm{h}=24$ & 0.52 & 0.32 & 0.60 & 0.01 & 0.17 & 0.26 & \\
\hline
\end{tabular}

Notes. Due to instability, the Diebold-Mariano $p$-values are not computed for the full sample. 
Table 3: Mean MSFE, relative to AR, by category

\begin{tabular}{|c|c|c|c|}
\hline \multicolumn{4}{|c|}{ FHLZ } \\
\hline & $h=6$ & $h=12$ & $h=24$ \\
\hline $\mathrm{IP}^{*}$ & 0.95 & 0.92 & 0.98 \\
\hline Employment & 1.13 & 1.07 & 0.98 \\
\hline Unemployment Rate & 0.87 & 0.91 & 0.94 \\
\hline Inventories & 1.00 & 0.93 & 0.98 \\
\hline Prices & 0.98 & 1.01 & 0.99 \\
\hline Wages & 0.98 & 0.99 & 0.99 \\
\hline Interest Rates & 0.99 & 0.98 & 0.95 \\
\hline Money & 0.87 & 0.86 & 0.75 \\
\hline Exchange Rates & 1.01 & 1.01 & 1.01 \\
\hline Stock Prices & 0.97 & 0.97 & 0.94 \\
\hline \multicolumn{4}{|c|}{ FHLR } \\
\hline & $h=6$ & $h=12$ & $h=24$ \\
\hline $\mathrm{IP}^{*}$ & 0.93 & 0.90 & 0.97 \\
\hline Employment & 0.98 & 0.95 & 0.91 \\
\hline Unemployment Rate & 0.67 & 0.72 & 0.85 \\
\hline Inventories & 0.95 & 0.89 & 0.97 \\
\hline Prices & 1.11 & 1.15 & 1.03 \\
\hline Wages & 1.08 & 1.05 & 0.97 \\
\hline Interest Rates & 1.12 & 1.14 & 1.07 \\
\hline Money & 0.90 & 0.93 & 0.79 \\
\hline Exchange Rates & 1.07 & 1.07 & 1.01 \\
\hline Stock Prices & 1.02 & 1.09 & 1.01 \\
\hline \multicolumn{4}{|c|}{ SW } \\
\hline & $h=6$ & $h=12$ & $h=24$ \\
\hline $\mathrm{IP}^{*}$ & 0.95 & 0.91 & 1.11 \\
\hline Employment & 0.94 & 0.94 & 1.00 \\
\hline Unemployment Rate & 0.68 & 0.74 & 0.97 \\
\hline Inventories & 1.04 & 0.97 & 1.24 \\
\hline Prices & 1.22 & 1.16 & 1.07 \\
\hline Wages & 1.15 & 1.12 & 1.12 \\
\hline Interest Rates & 1.32 & 1.52 & 1.55 \\
\hline Money & 0.98 & 0.94 & 0.88 \\
\hline Exchange Rates & 1.18 & 1.15 & 1.14 \\
\hline Stock Prices & 1.25 & 1.34 & 1.30 \\
\hline
\end{tabular}

Notes. MSFE, relative to AR, in the full sample, averaged over the variables belonging to each category (23 variables are excluded, see Section 4.2). The three factor models are compared. For $\mathrm{SW}$ the specification is $\mathrm{SW}_{2}(5)$. The best result for each horizon, over the three methods, in bold. IP* denotes the series belonging to Category 2, see Table ?? in Appendix ??. 
Table 4: Distribution MSFE relative to AR

\begin{tabular}{llllll}
\hline \multicolumn{5}{c}{ FHLZ } \\
\hline Percentile: & 0.05 & 0.25 & 0.50 & 0.75 & 0.95 \\
\hline$h=6$ & 0.82 & 0.92 & 0.99 & 1.02 & 1.22 \\
$h=12$ & 0.84 & 0.92 & 0.98 & 1.01 & 1.14 \\
$h=24$ & 0.82 & 0.94 & 0.98 & 1.01 & 1.07 \\
\hline
\end{tabular}

\begin{tabular}{llllll}
\hline \multicolumn{5}{c}{ FHLR } \\
\hline Percentile: & 0.05 & 0.25 & 0.50 & 0.75 & 0.95 \\
\hline$h=6$ & 0.64 & 0.93 & 1.04 & 1.12 & 1.18 \\
$h=12$ & 0.67 & 0.91 & 1.02 & 1.14 & 1.26 \\
$h=24$ & 0.77 & 0.90 & 0.98 & 1.06 & 1.21 \\
\hline
\end{tabular}

\begin{tabular}{llllll}
\hline \multicolumn{5}{c}{ SW } \\
\hline Percentile: & 0.05 & 0.25 & 0.50 & 0.75 & 0.95 \\
\hline$h=6$ & 0.65 & 0.95 & 1.12 & 1.23 & 1.38 \\
$h=12$ & 0.67 & 0.91 & 1.07 & 1.19 & 1.61 \\
$h=24$ & 0.73 & 0.96 & 1.07 & 1.29 & 1.63 \\
\hline
\end{tabular}

Notes. Distribution of MSFE, relative to AR, in the full sample, over 92 variables belonging to the dataset (like in Table 3, 23 variables are excluded, see Section 4.2). 
Figure 1: Instability of IP, CPI and the whole dataset in the Great Recession
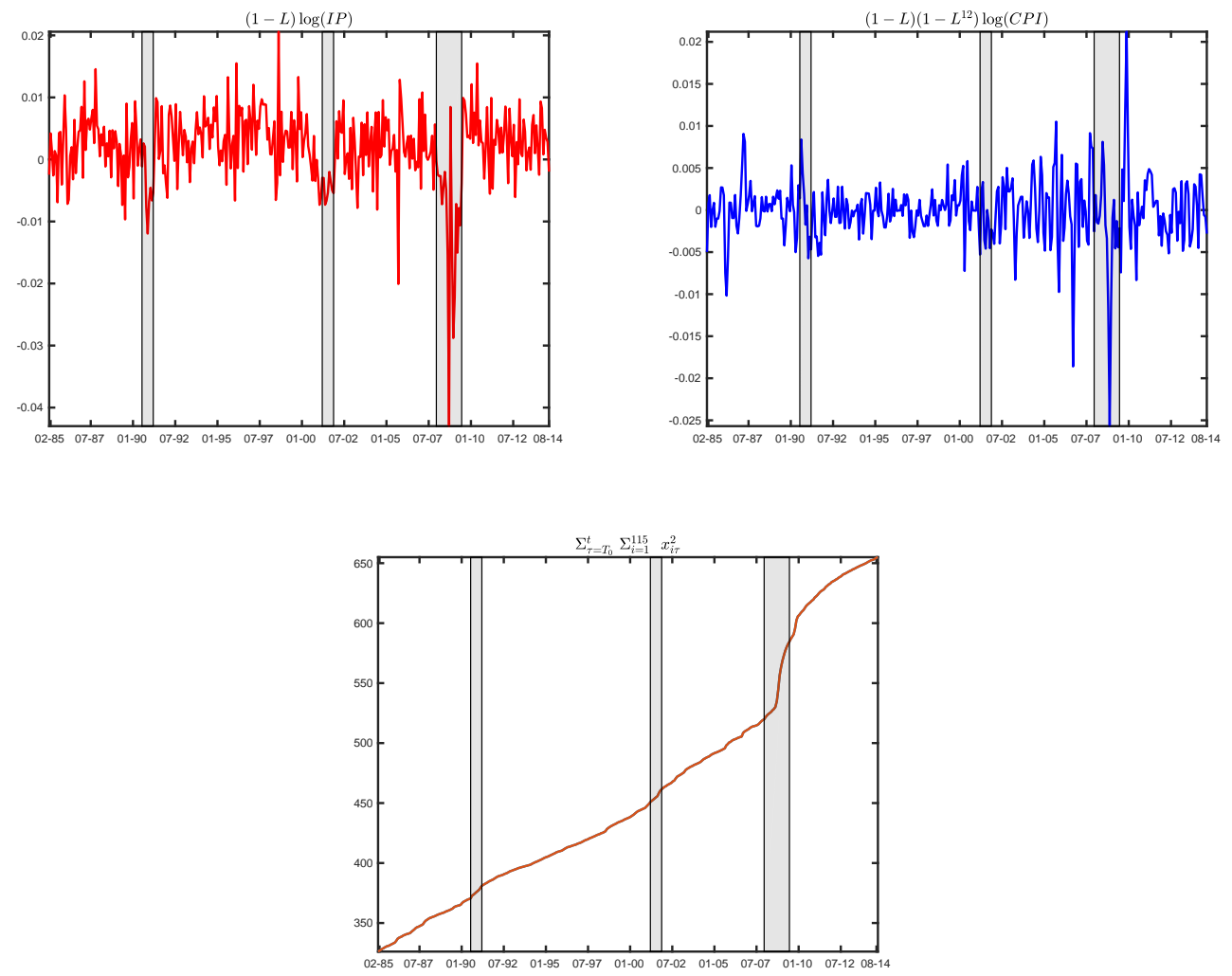

Notes. In the top graphs the variables $x_{1 t}=(1-L) \log \operatorname{IP}_{t}$ and $x_{77, t}=(1-L)(1-$ $\left.L^{12}\right) \log \mathrm{CPI}_{t}$ (recession periods in grey). Both show a marked departure from the stationarity assumption during the Great Recession. Rough evidence of the instability of the whole dataset is given in the lower graph, showing the cumulated sum of $\sum_{i} x_{i \tau}^{2}, i=1, \ldots, 115$, $\tau=1, \ldots, t$. 
Figure 2: Fluctuation test (IP)

FHLZ vs FHLR
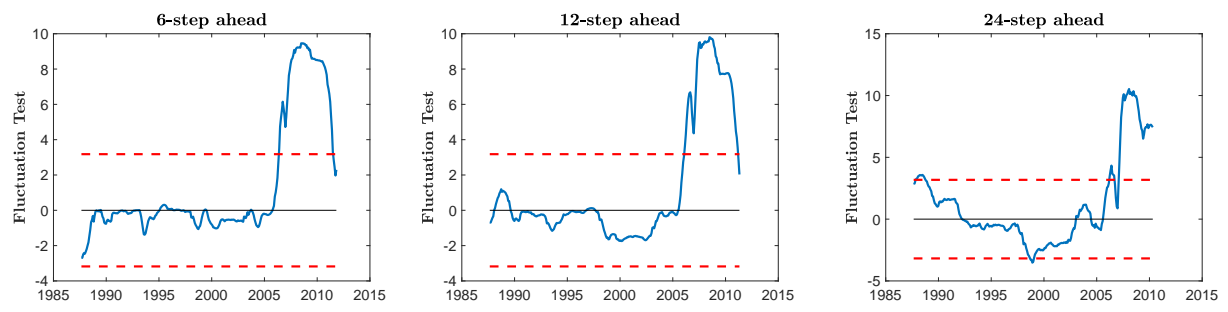

FHLZ vs SW
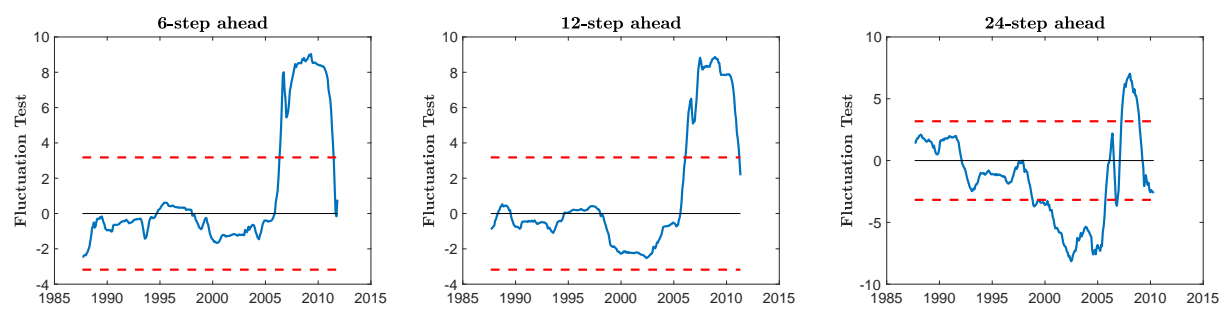

FHLZ vs AR
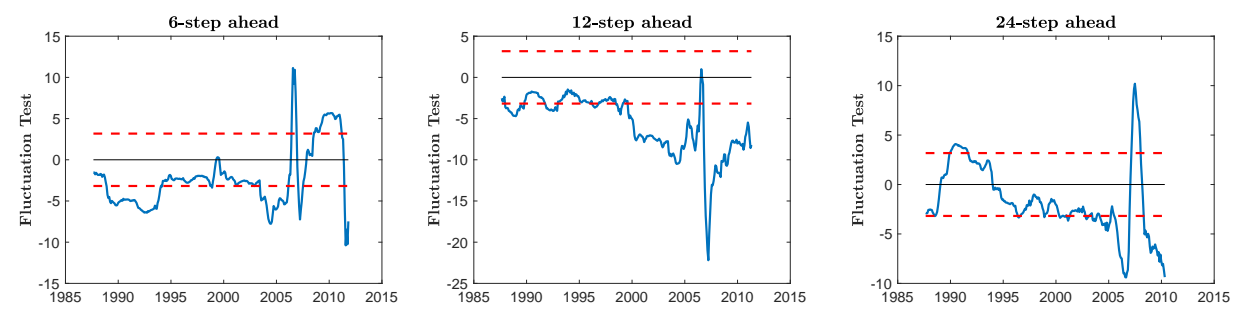

FHLR vs SW
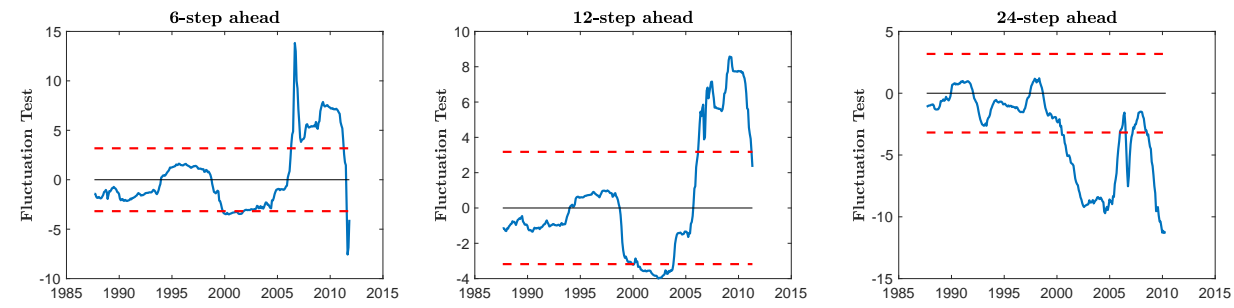

FHLR vs AR
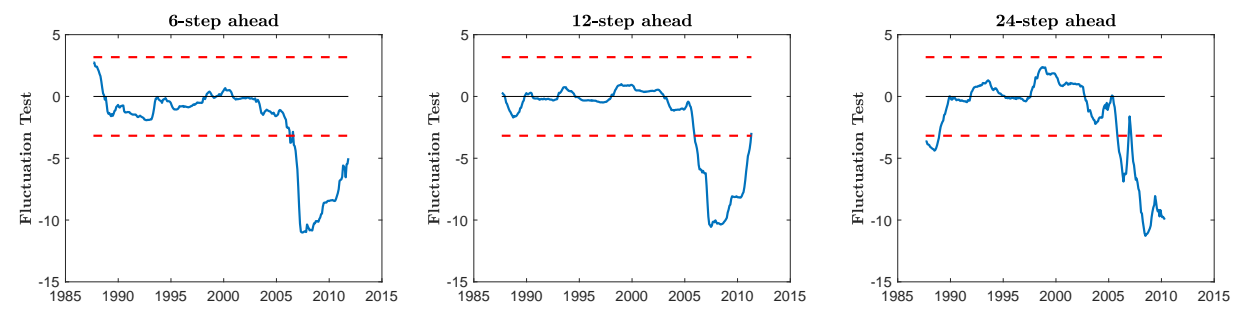

SW vs AR
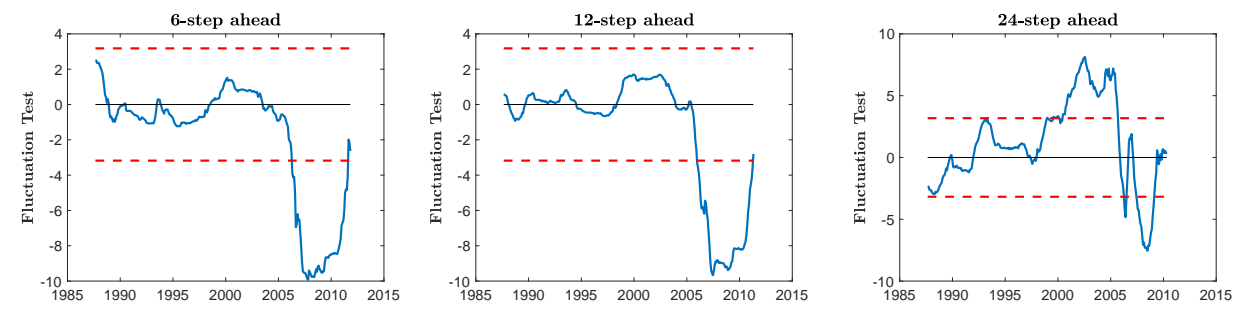

Notes. Fluctuation test statistic: Solid. $5 \%$ critical value: Dotted. If the solid is below the dotted (zero) line the first method is significantly better (better) than the second, and vice versa. 
Figure 3: Fluctuation test (CPI)

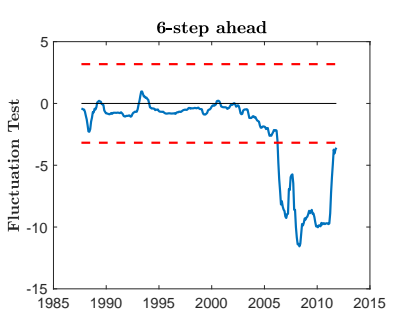

FHLZ vs FHLR
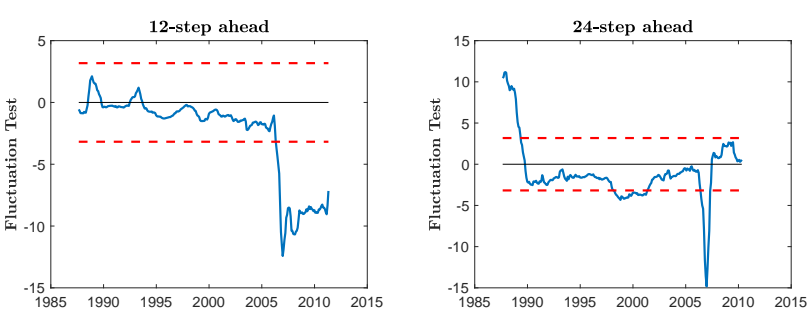

FHLZ vs SW
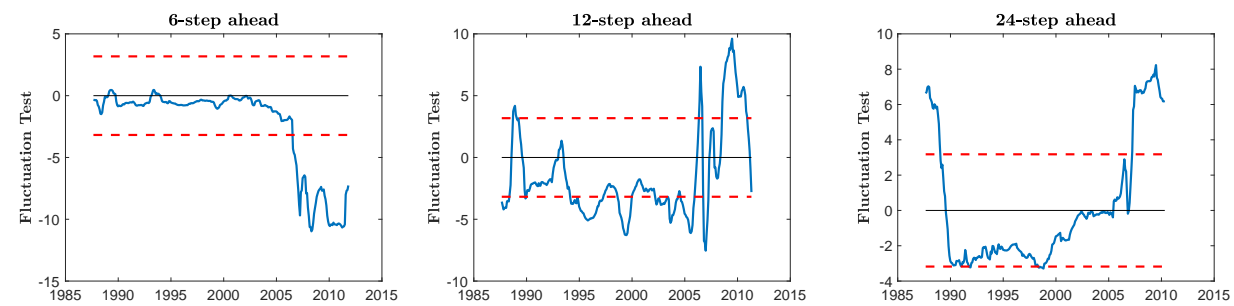

FHLZ vs AR
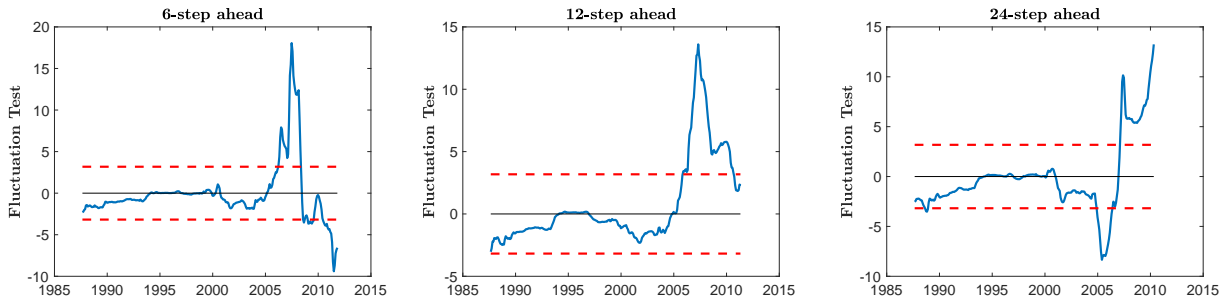

FHLR vs SW
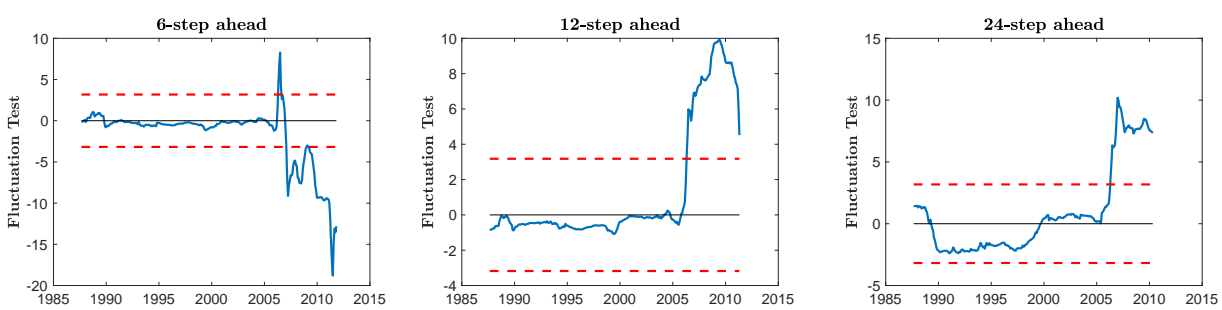

FHLR vs AR
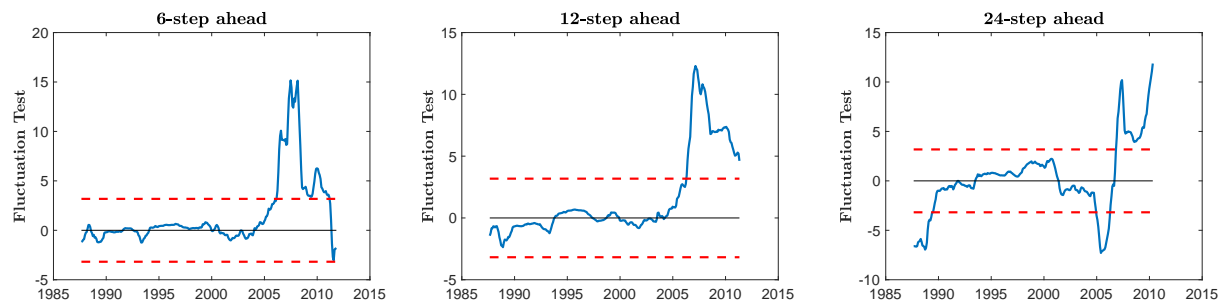

SW vs AR
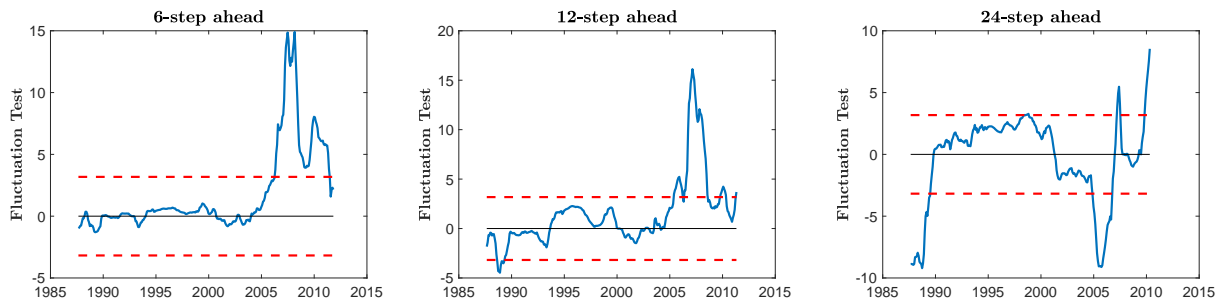

Notes. Fluctuation test statistic: Solid. $5 \%$ critical value: Dotted. If the solid is below the dotted (zero) line the first method is significantly better (better) than the second, and vice versa. 